\title{
Genres in Support of Collaborative Information Retrieval in the Virtual Library
}

\author{
Rob Procter and Ana Goldenberg \\ Department of Computer Science, Edinburgh University \\ Elisabeth Davenport, Department of Communication and Information Studies, \\ Queen Margaret College, Edinburgh
}

Andy McKinlay, Department of Psychology, Edinburgh University

\begin{abstract}
The advent of the digital -- or virtual -- library is usually presented as a positive development for library users. Much of the research and development work being carried out in this field tends to reinforce the perception of the use of information resources as a predominantly solitary activity, however. We argue that this narrow view of the virtual library may be counterproductive to its aims. Recent studies have emphasised that information retrieval (IR) in the conventional library is often a highly collaborative activity, involving library users' peers and experts in IR such as librarians. Failure to take this into account in the move to digitally-based resource discovery and access may result in users of the virtual library being disadvantaged through lack of timely and effective access to sources of assistance.

Our focus here is on the ways in which, in the conventional library setting, users consult with reference librarians for the resolution of their IR problems. We describe an investigation of consultation and collaboration issues as seen from the perspective of librarians' and users' contexts and analysed within the framework of genre. Drawing upon this analysis, we then describe the design of a prototype network, multimedia-based system which is intended to support collaboration between librarians and IR system users in the virtual library.
\end{abstract}

Keywords: information retrieval, collaboration, genre, virtual library

\section{Introduction}

The increasingly widespread use of on-line information retrieval (IR) systems represents for many people the first tentative steps in the realisation of the much heralded digital -- or virtual -- library. However, whilst the convenience of IR from the desktop is clearly very attractive for many library users, there are potential drawbacks to this development. For example, the opportunity that library users would normally have to consult with experts in IR to help resolve any problems may be seriously limited; if library users no longer visit libraries, it becomes important to consider how best to bring librarians' expertise to users' desktops.

Already, there is plentiful evidence of how valuable this expertise might be for users' success in online IR. Various studies show that users of OPACS (Online Public Access Catalogues) and other bibliographic databases encounter significant difficulties in finding the information they want (Borgman, 1996). These difficulties cannot be attributed to any single cause: factors include poor user interfaces, users' lack of knowledge of the domain, and poor search strategies (Marchionini, 1995). 
Whilst the case for better user interfaces for online IR systems is undeniable, concentrating on this issue alone fails to take into account the value of human expertise, and of collaboration, for effective IR. Recent studies of library users have shown how they frequently seek help from their peers with online IR problems (Twidale and Nichols, 1996), and that (though perhaps less often) they consult with domain experts such as reference librarians (Procter et al., 1996). Our interests lie in devising tools and environments for collaboration in the virtual library. We have examined more general issues of virtual library collaboration elsewhere (Procter at al, 1997). In this paper, we focus on collaboration between librarians and users, and consider how this might be better supported in the virtual library.

We begin by examining the reference consultation from the perspective of the traditional Library and Information Sciences (LIS) literature, and from that of more recent work on 'genre systems'. Then, in the light of such analyses, and of our own investigation of librarians and users, we consider the role of librarians as 'situated intermediaries' in users' IR tasks, whilst faced with multiple (and competing) demands on their time. Finally, drawing upon the results of our investigation and analysis, we describe work in progress to design and develop a prototype network, multimedia-based system to support consultation between reference experts and remotely-located users of bibliographic databases.

\section{Genres and Collaboration Between Users and Experts}

In the LIS literature, (collaborative) IR is closely identified with the reference interview, or reference interaction. Historically, this has been described as a question and answer session:

"... one of the most complex acts of human communication ... (in which) one person tries to describe for another person, not something he knows, but rather something he does not know." (Taylor, 1968).

Studies point to a framework of conventions that are observed within the reference interaction (e.g., Spink and Saracevic, 1993) in what may be termed 'classic' reference work. Such studies explain the reference interview as a series of 'communicative moves' both in the specific, micro-level context of the interview itself -- e.g., turn-taking between participants -- and at the broader, macro-level of the user's overall search goals. At this macro-level, Kuhlthau et al. (1992) have constructed a six-stage model of the search process: Initiation, Selection ("after a general area of inquiry is discerned"), Exploration ("when inconsistent and incompatible ideas are encountered"), Formulation ("when a focus is formed"), Collection, and Presentation, on the basis of analyses of transaction logs, verbal protocols, and qualitative studies of sense-making (Kuhlthau and Ledet, 1996). The phenomena of moves within typical reference interactions, and of observable stages in typical searches, have been noted in a number of empirical studies of user-online IR system interaction. Advice seeking and giving by means of the reference interview is manifest in some of these stages (e.g., Initiation; Exploration) more than others, as users draw upon the practices recommended by experts and other users, or suggested by documentary sources such as FAQ lists, to derive their own search heuristics. 
It has been argued that the gulf that exists between the micro- and macro-level accounts of search behaviour poses a major problem for IR as a discipline (Ingwersen, 1993), and recently there have been some attempts to bridge the two: Hert has taken this approach in her work on searching as 'situated action' (Hert, 1995). Whilst we also draw upon situated action, we suggest that exploration of the micro- and macrolevel contexts may be usefully subsumed under the label of genre analysis. Such an approach can provide a more comprehensive model of user-expert collaborations, and also provide a useful basis for the design of support systems for network reference consultation. One of the strengths of genre analysis for design is that it draws attention to the shared artefacts, and the media within which collaboration is embedded -- which are precisely those things over which the designer has control (Erickson, 1997).

A genre is a shared set of linguistic and structural features - socially constructed interpretive conventions - that guide both the production and consumption of communication (Brown and Duguid, 1994). It provides rules and resources to be deployed in the construction of a 'text' by actors within a specific set of social relations, or community (Yates and Sumner, 1997). In this way, genre serves to invoke a shared background and mutually intelligible behaviour within recurrent situations (Yates, Orlikowski and Rennecker, 1997). From the perspective of genre analysis, Kuhlthau's search process stages may be compared with situations, and moves with behaviours triggered by the situation's characteristic cues. For example, the reference interview may be interpreted as a genre invoked by the librarian as a response to a query from a user at a particular stage of a search. As we note above, however, a reference interview will not necessarily be an appropriate response for the librarian to make at every stage of a search. As we shall see later, librarians' need to be able to manage the various demands on their time is a major reason why they call upon a repertoire of responses, e.g., referral to documentation.

Genres are enacted through media; genre and media are therefore distinct, but “... media may be a salient aspect of a particular genre's form." (Yates and Orlikowski, 1994: $p$ 133). The successful enactment of spoken genres, such as the classic face-toface reference interview, relies upon participants' capacity to reproduce the appropriate linguistic and structural features. In other media, however, features of the genre may be embodied within the media itself. For example, the design of the office memo (its fields, layout, size etc.) serves to reinforce its purpose for both producer and consumer. Within any particular setting, several distinct genres will typically be in evidence and, more often than not, can themselves be observed being employed in recurring patterns. Such 'genre systems' can be defined as “... an interrelated sequence of genres enacted by the members of a particular community ... composed of a well-coordinated set of communicative moves that together accomplish an interaction ... the system as a whole, as well as the individual genres constituting the system, can be said to have a socially recognized purpose and common characteristics of form." (Yates, Orlikowski and Rennecker, 1997). Thus, the memo which notifies group members about a forthcoming meeting, the agenda, papers and minutes (once the meeting has taken place), collectively constitute a genre system. By analogy, the reference consultation -- a request for help in a subject area search, the ensuing iterative process of display of results and feedback from the user, and the delivery of the final document set -- may itself be intrepreted as constituting a genre system. 
Genre analysis has demonstrated its value as a tool for understanding how organisational actors use new electronic media over time, and how such use influences communicative practices (Orlikowski and Yates, 1994). It has also been used to analyse communication beyond conventional organisation boundaries, for example, in communities on the World Wide Web (Crowston and Williams, 1997). Erickson (1997), in discussing genre analysis for online discourse, suggests a focus on:

- the nature of the discourse community;

- the communicative purpose of the discourse;

- the regularities of form and content of the communication, and the underlying expectation and conventions; and

- the properties of the recurrent situations in which the genre is employed, including the institutional, technological, and social forces that give rise to the regularities of the discourse.

Reference consultation may be described in terms of these four focal areas. Its 'discourse community' is that of library users and experts, the 'purpose' and 'form' are the provision of information within the institution of the library, and the 'regularities of the discourse' are the communicative moves which characterise (for example) the reference interaction. As we have seen, however, the classic reference interview is but one of several genres observed within the consultation process. For example, for users of an online IR system, recommended practice will also be articulated through document-based genres such as online and printed manuals. Alternatively, users may choose to proceed on the basis of experience gained with other systems, or through discussions with their peers. In addition, the classic reference interview takes for granted circumstances which may not be available or reproducible within the virtual library environment. The aim of our investigation is not simply to try to reproduce the circumstances of the classic reference interview in networked collaborative reference work: rather we are interested in what variants of existing -- or even new -- genres may prove useful, either independently or as constituent elements of enhanced genre systems, in this novel setting. As we indicate below, our preliminary investigations of the work patterns of experts and users suggest that 'variants' are more in evidence than the classic reference interview.

\section{The Investigation}

We have used a variety of techniques to build up a picture of forms of collaboration between reference librarians and library users in the contemporary academic library, and of the 'reference consultation' in particular. These included interviews with librarians, questionnaires distributed by email and the WWW to a target group of around 150 on-line bibliographic IR system users, and ethnographic-based observations of interactions between library users and librarians. Librarians at Edinburgh, Heriot-Watt and Napier Universities took part in the study. The investigation's scope included:

- classifying the problems users typically encounter;

- analysing how problems and solutions are articulated, including the rules which operate within reference consultations; and 
- the factors which circumscribe reference consultations and shape librarians' practices.

The initial service focus for the investigation was the BIOSIS abstracts database, recently introduced as a networked reference resource, and available to participant institutions via the Edinburgh and Stirling Metropolitan Area Network (EaStMAN). Our exploration of the issues, however, has subsequently led us to broaden the focus of the inquiry to consider the BIOSIS work (or reference consultation) as just one component of a reference librarian's remit. We now present and discuss in turn the results of our investigation as they relate to librarians, IR system users and genres for their collaboration.

\section{The Library Community}

Overall, our observations of those librarians involved in BIOSIS user support provide a picture of reference consultation work which is very different from that suggested by the classic reference interview of the LIS literature. Each participant librarian was asked to keep a diary for one complete week at different stages of the investigation. These indicate that the librarians are subject to a barrage of miscellaneous and heterogeneous questions, from users whom they do not know, in a pattern of consultation which is characterised by high volume, impersonal, uninformed, and short exchanges.

The dearth of sustained reference consultations reflects the fact that fewer such demands are made of reference librarians in a world where end-users already tend to serve themselves. The rise of autonomous searching by end-users has been mapped in a series of longitudinal studies by East and his colleagues (East, Sheppard and Yeal, 1995). The comments of East's interviewees are echoed in those of two librarians in our study who stated that after induction or training sessions on a given database, “... users just disappear; we don't know where they go". Another librarian commented:

"We have library [WWW] pages which we use to communicate news and services. But it depends how often people look at it. You could see how many times a page has been visited, but it doesn't give us any indication of who's been looking at it. I wonder whether they all know about it really."

Interviews with librarians also addressed the context, from the librarian's perspective, in which these consultations take place. All described full schedules (confirmed under observation and by the diaries), with nomadic work patterns which involved multi-tasking at least some of the time (answering a phone query, for example, while working with paper, or dealing with email; cataloguing, online searching and dealing with verbal enquiries at the reference desk), with the result that ground has to be cleared before the communication moves that characterise classic reference interaction are brought into play. Our observations of reference work showed that where sustained consultation occurs of the sort described in the LIS literature, with face-toface interaction over a workstation and finessing of feedback with a user, there are observable interaction patterns which can be attributed to the librarian's professional training. These may relate to the librarian's perceptions of the stage of the search, perceptions of the user's expertise (i.e., competence in encoding the search in terms of 
formal search statements), or interpretation of 'user needs' which may guide the librarian in decoding (assessing the relevance) of retrieved material. Such actions, which constitute the classic reference consultation, may be described, as we have argued above, in terms of a genre system.

In many cases, however, the demands made of librarians are not amenable to treatment by means of rule-based consultations that relate to a subject area: users' demands are not only concerned with content, but with technical or other kinds of problem. The diaries also clearly show that few of the librarians carry out extensive reference consultation. Most of the collaborations logged in diary records are short (three to five minutes) interactions of the 'help desk' kind, rather than subject searches, and follow a pattern of multiple tracking of user needs rather than dedicated, serial reference interactions. The content of such consultations involves multiple 'starting overs' rather than sustained 'problem solving' reference work (Taylor, 1968).

In these situations, librarians appear to operate within a framework of appropriate responses to cues: sets of criteria for appropriate behaviour are also brought into play. For example, some librarians prioritise responses on the basis of communication media: face-to-face has precedence over the telephone, and the telephone has precedence over email. Other criteria for managing multiple demands on time would seem to be based on, inter alia, the status of the user, a hierarchy of perceived user needs, and the level of skill required of an expert who attempts to satisfy them. Where such criteria allow librarians to identify areas where expert intervention is not appropriate (e.g., low-level requests for technical help), it enables them to make a judgement about how to use their time effectively. As we shall see later, it is equally important that users be aware of such criteria so that they entertain appropriate expectations of librarians and their availability: the successful enactment of genre requires that participants make mutually compatible judgements.

\section{The User Community}

A user questionnaire was designed to help build up a broad picture of usage, users' views of the BIOSIS system, and to develop a picture of the kinds of problems that were typically encountered. Over a period of 6 months, a total of 38 questionnaires were returned. The largest group of respondents were academic staff (30\%), followed by postgraduate students (24\%), undergraduate students (20\%), research staff (14\%) and finally miscellaneous library and information services staff (12\%). As a sample, academics and students are clearly unrepresentative of the wider online IR system user population: the majority (56\%) are in occupations where the ability to find and utilise information is an essential skill; of the student users, only the undergraduates (20\%) might justifiably be considered as relatively unskilled and/or inexperienced IR system users. The results should not be taken as necessarily typical in either the frequency of occurrence or type of problems; in fact, given our sample's relatively high levels of skill, the results may be an underestimate of the difficulties encountered by the IR user population at large. If this is indeed the case, then it strengthens the argument for trying to improve users' access to IR expertise.

Users were asked how successful they thought they had been when using the BIOSIS system. Only $2 \%$ replied that they had been unable to find anything they were looking for, whereas $44 \%$ replied that they either found exactly what they wanted or a 
lot of relevant information. However, this positive picture is somewhat diminished by the $36 \%$ of replies from users who stated that they only found some useful information. Users were also asked questions regarding their competence in performing searches. The majority of respondents - $61 \%$ - stated that they judged themselves as either competent or very competent at performing complex searches; however, a significant minority - 39\% - replied that they did not consider themselves competent in this respect. As anticipated, the majority of undergraduates placed themselves in this latter group. Problems reported ranged from the technically trivial - but practically significant -- (e.g., forgotten passwords), to procedural (e.g., command language syntax) and conceptual problems (e.g., compound searches).

The final part of the questionnaire was designed to gather users' opinions and expectations of the different ways of dealing with the problems they encounter when using the IR system. Specifically, users were asked to rate various combinations of sources of expertise and means of access according to their:

1. effectiveness in providing assistance; and

2. convenience, i.e., responsiveness and ease of access.

The sources of assistance were Librarian, (another) BIOSIS user, Colleague and FAQ list (as a form of online help), which were chosen to reflect the range of expertise that might normally be expected to be available to a user. The results thus would not only enable us to gain a picture of the value BIOSIS users place on different kinds of expertise, but also an understanding of how they respond to the circumstances in which access to the expertise is embedded.

The methods of access were synchronous, unmediated speech (face-to-face), synchronous, mediated speech (telephone), and asynchronous text communication (email). These were chosen because they are commonly used, and also because they can be mapped onto distinct categories of the familiar Computer-Supported Cooperative Work (CSCW) classification of media. Thus, face-to-face exemplifies a same time + same place media, telephone exemplifies a same time + different place medium, email exemplifies a different time + different place medium, and FAQ list (as an archive summary of past consultations with a common point of access) is an example of a genre based upon a different time + same place medium.

The results are summarised in Figures 1 and $2(-2=$ not very effective/convenient, +2 $=$ very effective/convenient). Consider first of all the respondents' assessment of the effectiveness of the different sources of expertise face-to-face (see Figure 1). For comparison, users' assessment of FAQ list effectiveness is also shown. It would be surprising if Librarian was not ranked highest, and this is borne out by the results. Librarian is followed in order of decreasing effectiveness by BIOSIS user and Colleague. Again, this is broadly in line with expectations: a BIOSIS user is more likely to have relevant expertise and experience than the typical work colleague. What is interesting, however, is the high rating given to FAQ list: overall, only Librarian is rated more highly. We conclude that this reflects a recognition of the underlying similarity of problems encountered within the user community. There were no significant differences in effectiveness ratings between the two major subgroups within the user sample (academic staff and students). 


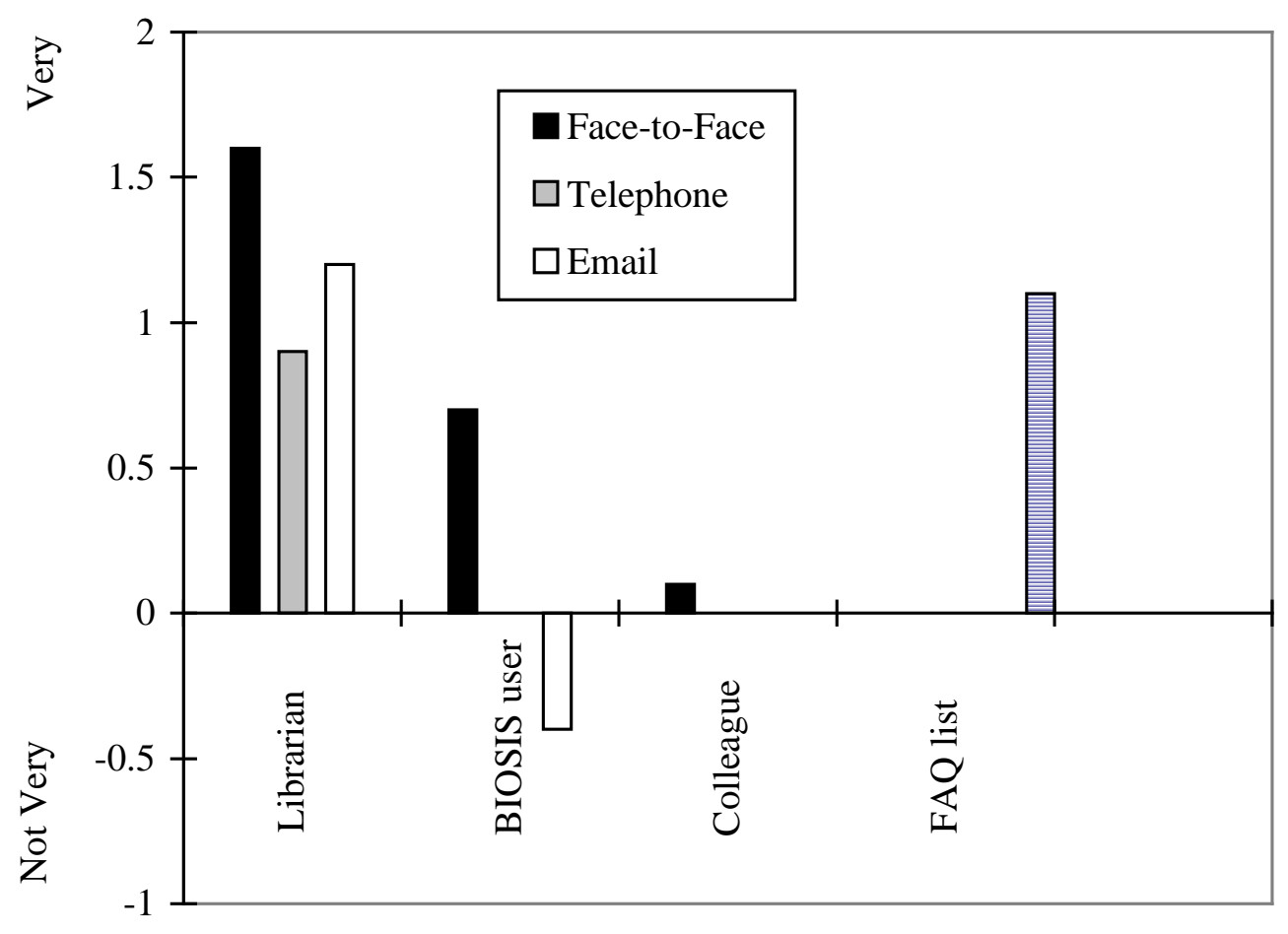

Figure 1: Users' rating of effectiveness of expertise source by medium.

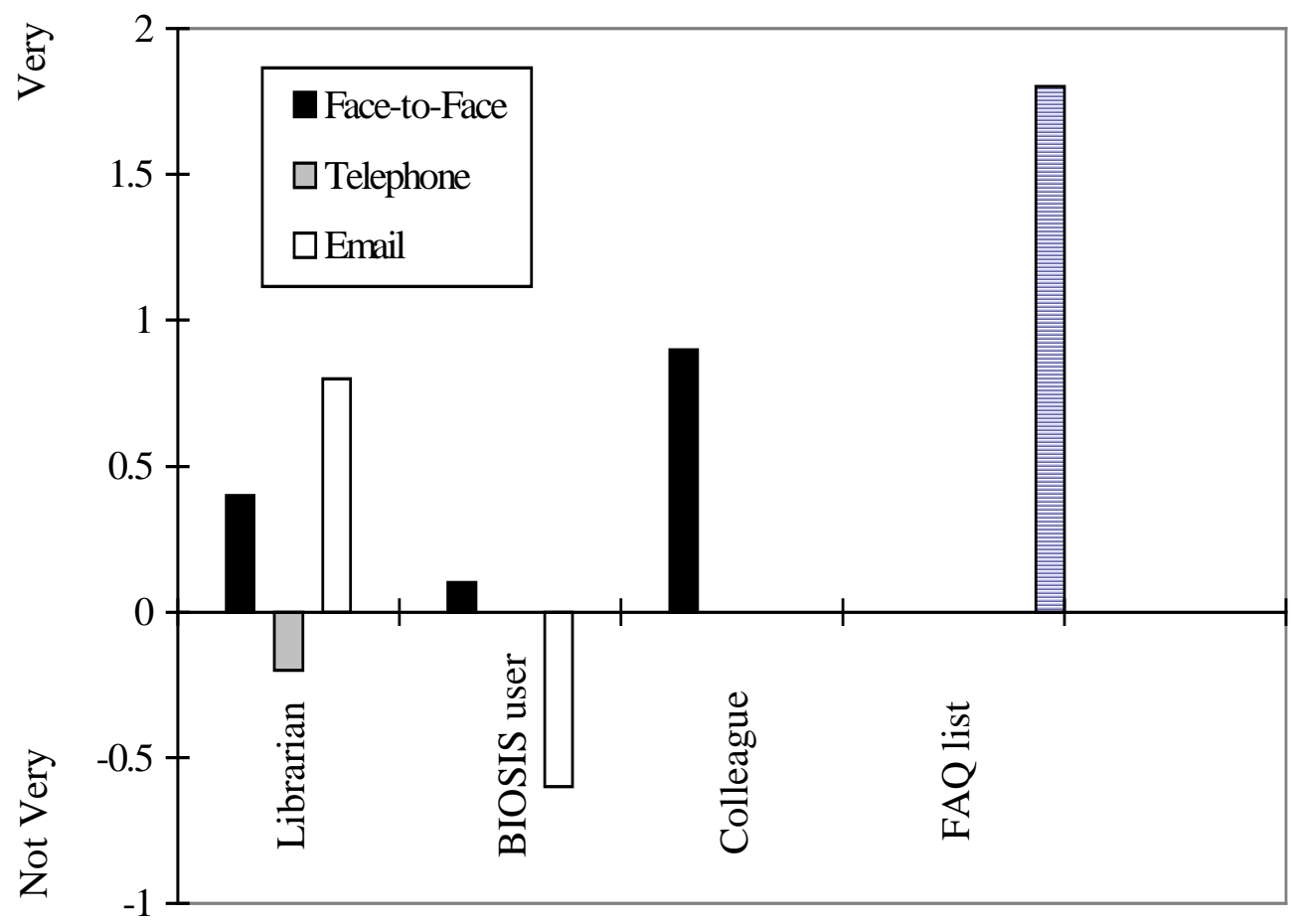

Figure 2: Users' ratings of convenience of expertise source by medium. 
Figure 1 also compares respondents' ratings of the effectiveness face-to-face with telephone and email. Again, it would be surprising if face-to-face were not rated more highly than both telephone or email, and this is confirmed by the results. The preference expressed for face-to-face may be due to several factors, including artefact sharing (e.g., sharing printouts, pointing to a screen) (McCarthy, Miles and Monk, 1991). This, we argue, should be conserved in network reference work.

Consider next the convenience rankings for the three human sources of expertise delivered face-to-face, together with FAQ list (see Figure 2). Here, we find that FAQ list is (as expected) rated most highly, followed by, in decreasing order: Colleague, Librarian and BIOSIS user. In contrast with the effectiveness ratings, there were also significant differences between the respondent sub-groups on this measure: in particular, academic staff rated consulting with a Librarian face-to-face as the least convenient method, whereas students rated this category as second only to FAQ list. We argue that the explanation for these results lies in the role of place and its effect on the accessibility of human expertise. Academic staff are more likely to use BIOSIS from their offices, whereas students most often use BIOSIS from public terminals situated within the library: it is inevitable that the latter will find librarians more accessible face-to-face. In these results we find a simple, but clear acknowledgement of the disadvantages of IR from the user's desk top: one question is whether other media such as telephone and email are perceived by BIOSIS users to provide an effective alternative to visiting the library.

Figure 2 also compares survey respondents' assessment of the convenience of faceface with those of the other media. For Librarian, email was the most highly rated, and telephone the least highly rated, with face-to-face coming somewhere in between. One explanation for this may lie in users' experience of the work patterns of the experts: as we have seen, these mean that experts' availability may be a very hit and miss affair. Another might be users' lack of understanding of an expert's assessment of what is an appropriate response, e.g., because users are not fully aware of how librarians prioritise requests for assistance. The case of BIOSIS user is interesting in that it presents a different picture of peer collaboration to that found in studies of the wider library user population (e.g., Twidale and Nichols, 1996). BIOSIS users don't expect to encounter other BIOSIS users face-to-face, nor do they have very high expectations of network encounters by email. These results suggest that, in comparison with the wider library user population, BIOSIS users' informal support networks are relatively weak; the BIOSIS user community inhabits a virtual environment; it is dispersed and its members are largely unknown to one another. Some corroboration for this view is evident in the especially low rating for consulting a BIOSIS user by email: whereas email is rated as more convenient than face-to-face for communicating with Librarian, the reverse is true for a BIOSIS user.

\section{Implications for Design}

Our investigation provides evidence for how unwanted consequences may follow from adopting network technologies without taking into account the wider circumstances of the work setting. More specifically, the picture which emerges is of a community whose membership (both librarians and users) are increasingly anonymous, a situation which, if not directly attributable to the growth of online, 
networked information services, is exacerbated by them. We can see clearly the effect of the absence of a clearly defined social space in which a community's members can congregate, and make themselves and their activities publically available to one another. For librarians, one result is that the overheads of starting up a consultation -establishing the problem's common ground -- are increased. For BIOSIS users, the lack of an identifiable community makes it more difficult for them to seek assistance from each other. As Erickson observes, the attributes of conventional social spaces are not easily reproduced in their virtual equivalents (Erickson, 1997). By way of addressing this, we argue that the design of a system to support network reference consultation should acknowledge:

- the cues which operate within consultations;

- the situations which condition them or shape the practices within which consultations occur; and

- the matching of genres, including media-based artefacts to cues and situations.

The kinds of communication resources provided should reflect the variety of forms of interaction and collaboration genres which arise as a result of the interplay of the above factors. Our investigation leads to three specific design recommendations.

First, librarians' time is in short supply and their capacity to take part in sustained, synchronous interactions is severely constrained by the multiplicity of tasks in which they engage during the working day. They might find it very difficult if the network consultation service denied them the power to control their accessibility and manage their time. In these circumstances, asynchronous media-based genres are likely to play a particularly important role. Reference librarians might benefit from novel genre-based approaches which would enable them to 'fast track' into a relationship with the user. For example, Twidale and Nichols (1996) have explored graphical representations of user-IR system interaction as a way of providing a view of user search moves which can be interpreted at a glance, or 'instant client history'.

Second, both librarians and users would benefit from being able to adapt genre systems to match the changing needs of the ongoing consultation process, without sacrificing access to relevant artefacts such as queries and their results. This emphasises the importance of the integration of media and artefacts within the network reference consultation environment.

Finally, it is important to encourage the peer collaboration evident in the conventional library space, e.g., by making the user community's collective knowledge and experience more easily available. In this context, resources such as FAQ lists are a particularly valuable low-cost genre for the accumulation of information 'capital' and for making it accessible on a community-wide basis. Indeed, our survey shows that users rate FAQ lists relatively highly as a resource for resolving their IR problems. 


\section{A Prototype Network Reference Consultation Support System}

A prototype network reference consultation support system is being implemented using various digital media, including video, audio and text conferencing, and email. The system is implemented as a WWW-based server and requires only a WWW browser for access ${ }^{1}$. In designing the prototype, we have applied the results of our investigation to establish possible mappings between the various available media and artefacts, and to integrate them within appropriately defined genres for network reference consultations. An outline of the prototype system architecture is shown in Figure 3.

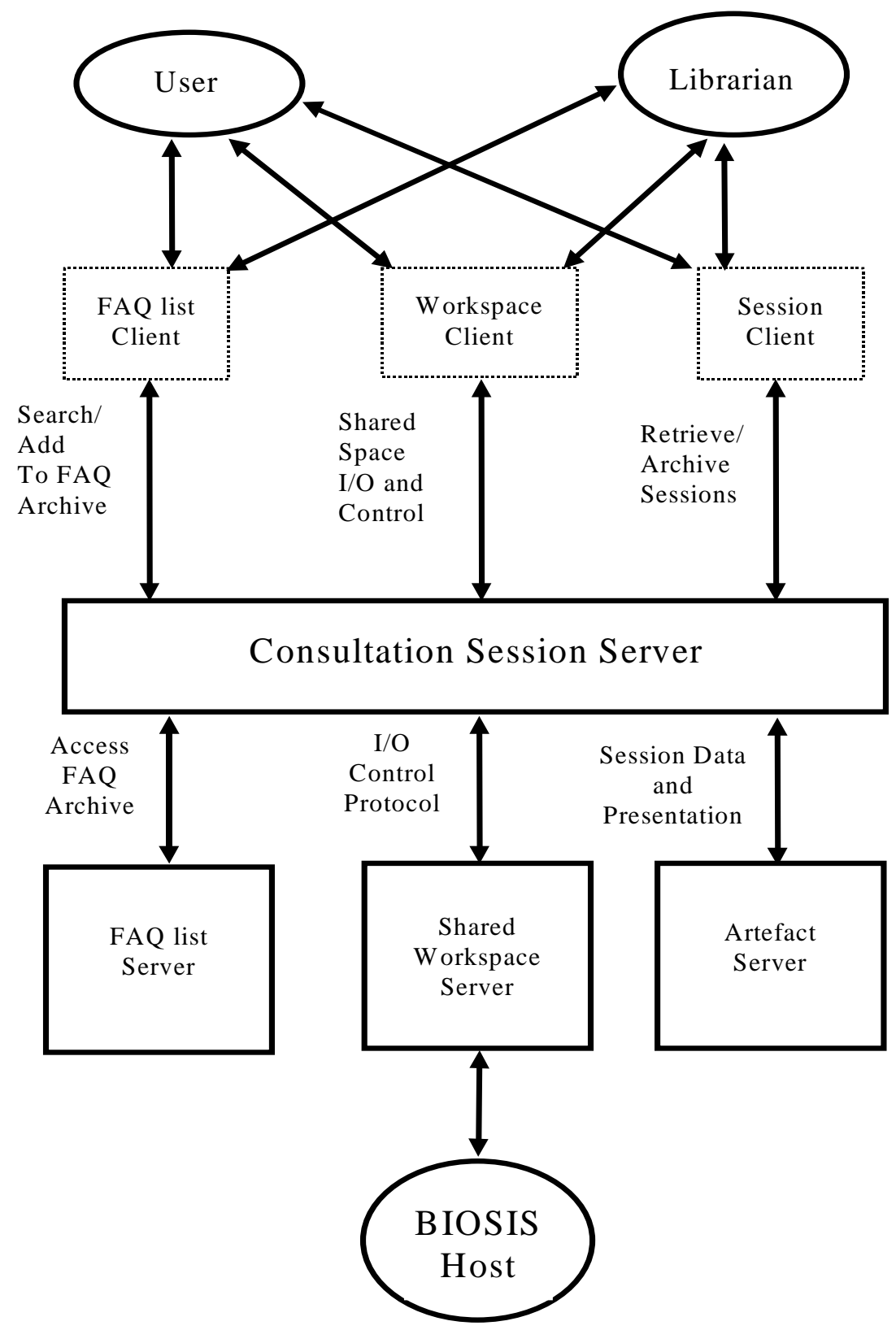

Figure 3: Network Reference Consultation server architecture.

\footnotetext{
${ }^{1}$ http://www.remote.lib.ed.ac.uk
} 
The system architecture is based on a client/server model. There are three main types of clients and servers (see Figure 3). On the client side we have: Workspace Client, a Java Applet that manages the individual shared working space, and deals with all controls related to the shared workspace, such as turn-taking; Session Client, a Java Applet that manages the individual IR sessions, and stores them in MIME compatible files so that they may be passed around as attachments, or accessed from the FAQ archive through WWW browser; and FAQ list Client, a Java Applet that allows a user to navigate through the FAQ archive, and to add information into it. On the server side we have: Workspace Server, responsible for establishing the connection between all Workspace Clients and their destination hosts, as well as establishing and controlling the sharing between users and librarians; Artefact Server, responsible for archiving and retrieving users' online IR sessions; and FAQ server, responsible for managing the FAQ archive.

\section{Media and Genres for Collaboration}

Participants in genres enacted face-to-face (e.g., an interview or a consultation) are able to draw upon a rich variety of verbal and non-verbal contextual cues which make a significant contribution to communication structure and management. As stated earlier, the shared, physical work context that a face-to-face genre affords is also important for establishing and sustaining common ground with the minimum of effort (McCarthy, Miles and Monk, 1991). One important issue for the design of the network reference consultation system is the extent to which the full circumstances of a face-to-face encounter need be recreated for the effective enactment of a reference interview via alternative media. Both laboratory and real-world studies suggest that audio is the most important media for efficient synchronous, network-mediated collaborations (Gale, 1990), but that participants often express a strong subjective preference for video (Olson, 1995).

Video-conferencing represents the closest practical recreation of the face-to-face encounter currently available. Initial trials with volunteers indicated that untutored users find the cheaper LAN-based systems (e.g., CUSeeMe) difficult to use effectively (Procter et al., 1996). These same trials also suggested, however, that there may be a role for video-conferencing in peer-to-peer collaboration between librarians. In the prototype system, synchronous consultations will be supported through audio- and text-based conferencing, with common ground provided through a workspace sharing tool. The latter enables user and librarian to jointly observe and interact with the user's current online IR session and to retrieve and discuss past IR sessions and FAQ list items. An example of a synchronous text-based consultation is shown in Figure 4.

WWW-based tools are also being developed for the project to support asynchronous consultations, including the sharing of bibliographic search histories, and archive management (e.g., the automatic generation of FAQ lists, and FAQ list-like search histories, from the flow of queries and answers). The latter is based upon AnswerWeb, a WWW-based implementation of Answer Garden (Ackerman and MacDonald, 1996). An example of an asynchronous consultation via email is shown in Figure 5. Here, a user is about to send an email query to a librarian, with an attachment showing the context of the problem. 


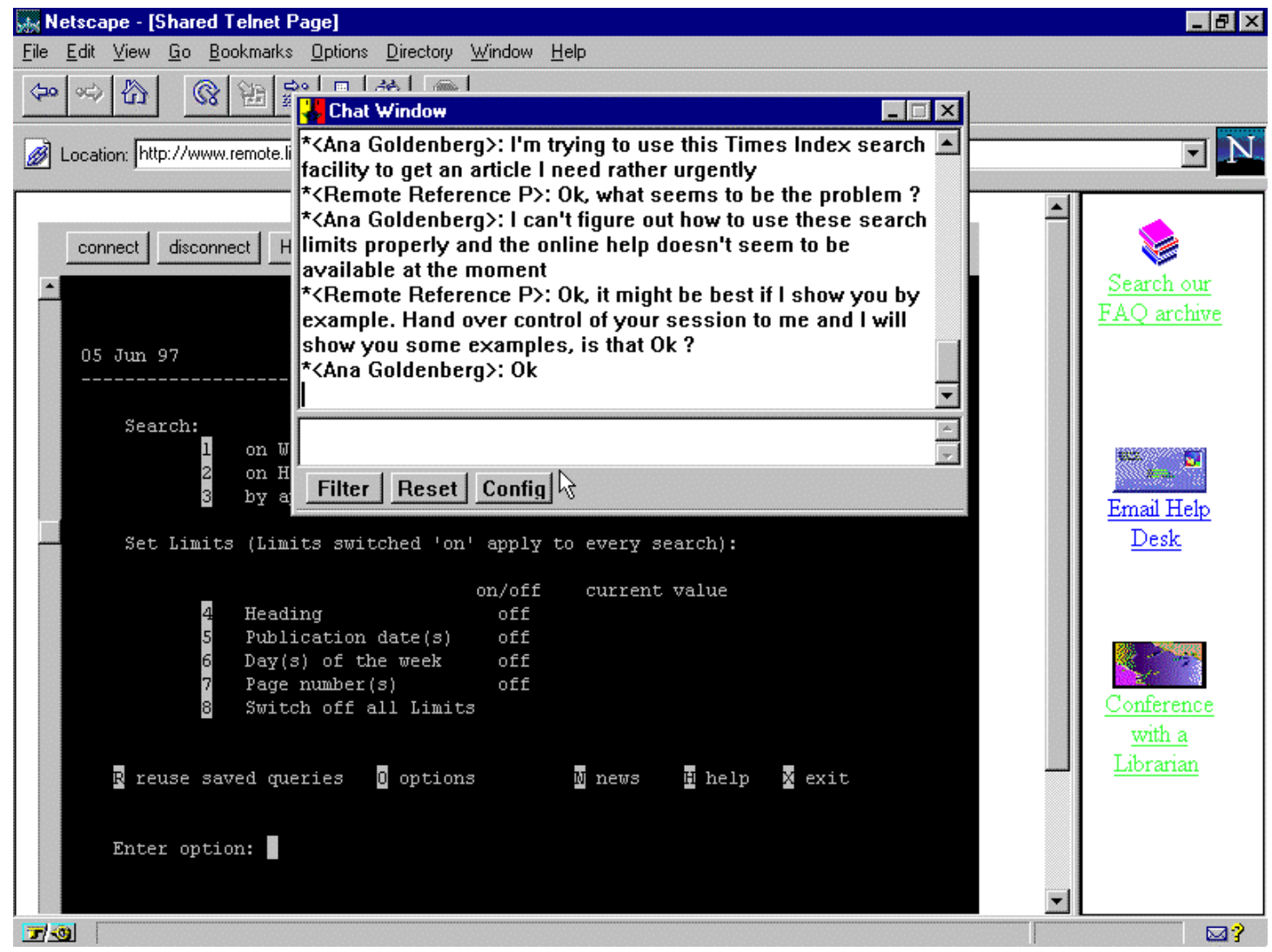

Figure 4: Prototype synchronous text reference consultation tool.

The integrated architecture of the system (see Figure 3) is designed with the goal of enabling both user and librarian to identify and apply appropriate genre and media for queries and responses, including the 'graceful escalation' of queries, within the framework of a particular genre system.

\section{A Framework for Genre Analysis and Situated Reference Work}

The emergence of genres can be linked to theories of situated action which have offered explanatory frameworks for both organisational and educational research. Suchman's version of the concept -- and the methods associated with it (direct observation and analysis of verbal protocols) -- emphasises organisational ethnology and the emergence of strategies to handle recurrent events, and is relevant to our investigation. Equally relevant are the observations that lie behind 'situated learning' (Lave and Wenger, 1991; Brown and Duguid, 1993) where learner and pedagogue (a ready analogy can be made with user and expert librarian) seek to establish comparable understandings of each one's expectations of the other. Situated learning draws on the "ordinary, everyday, finely detailed methodic practices of participants to an activity in specific settings" (Fleming, 1994: p. 525) and learning, in this context, means being able to participate appropriately in the settings ... "where the subject or discipline is being done" (op cit: p. 526). Situated intermediation, by analogy, means "being able to participate appropriately in the settings ... where questions are being asked". In the traditional library, the burden of learning about 'appropriate participation' has fallen more heavily on users who are expected to undergo 'library 


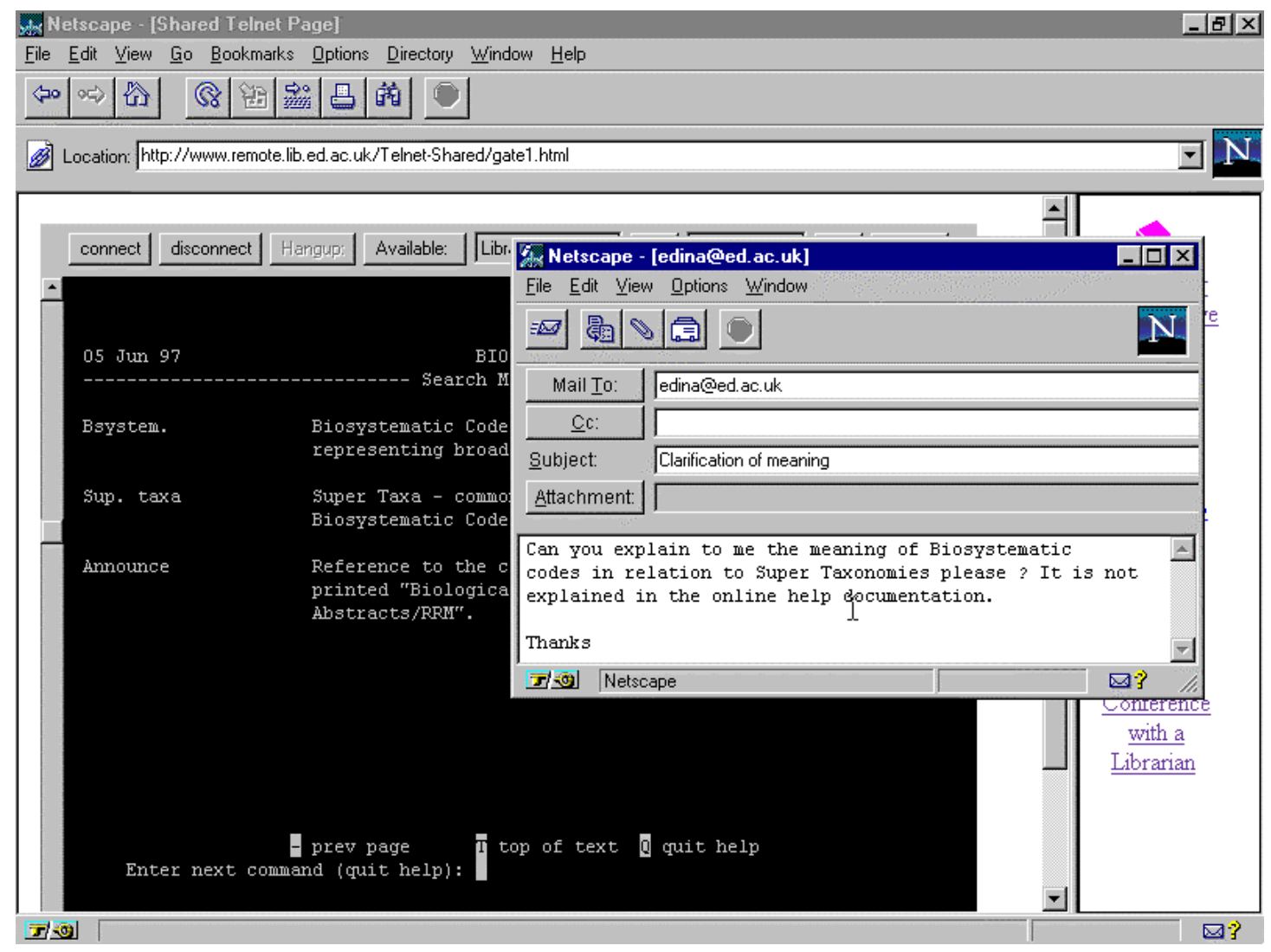

Figure 5: Prototype email consultation tool.

induction' in order to make effective use of the system. In a remote reference consultation setting, the onus will be on the librarian, who has to figure out what is happening at the user's desktop. The notion of appropriate participation is further complicated by the implosion of tasks in the library: 'settings' in the case of our participating librarians go beyond the bibliographic database of classic reference work, to include any type of interaction with the institution, and not all of these settings may be the province of the professional librarian.

We will be using the prototype system to support a pilot network consultation service, and this will allow us to assess the relationship between genre and situation. In addition to the framework provided by Erickson, we are using indicators from situated learning to investigate this relationship further. Fleming suggests the following steps (Fleming, 1994):

- a structural anatomy -- how are sequences assembled and constructed in the specific settings in which they are used;

- a functional anatomy -- the use to which methodic practices are put on a given occasion; and

- an understanding of the machinery by which such activity works -- how do descriptions, facts, processes work together to produce what participants in the dialogue recognize as an explanation of the phenomenon in question? 
We can infer from existing BIOSIS user group logs, and from the diaries of library reference help desks, that there will be at least three genre systems: the reference interaction; the help desk (we are currently exploring an email archive in this context); and peer assistance (Procter et al., 1996). As the archive of pilot service use grows, we may see the emergence of genre systems that relate to the archive itself, e.g., the refinement of FAQ lists etc. Some possible genre systems are illustrated in Table 1, with each row representing a specific example.

\begin{tabular}{|c|c|c|c|}
\hline Situation & Cues & Appropriate response & Genre \\
\hline User can't log on & $\begin{array}{l}\text { Query on } \\
\text { procedure }\end{array}$ & $\begin{array}{ll}\text { - } & \text { FAQ } \\
\text { - } & \text { Online help menu } \\
\text { - } & \text { Expert referral } \\
\end{array}$ & $\begin{array}{ll}\text { - } & \text { FAQ list } \\
\text { - } & \text { Online manual } \\
\text { - } & \text { Email } \\
\end{array}$ \\
\hline $\begin{array}{l}\text { User wants material } \\
\text { for an assignment } \\
\text { (Initiation phase) }\end{array}$ & $\begin{array}{l}\text { Query on subject } \\
\text { area sources }\end{array}$ & $\begin{array}{ll}\text { - } & \text { Online help menu } \\
\text { - } & \text { Expert referral } \\
\text { - } & \text { Reference interview }\end{array}$ & $\begin{array}{ll}\text { - } & \text { Online manual } \\
\text { - } & \text { Email } \\
\text { - } & \text { Text/Audio/Video } \\
& \text { conference } \\
\end{array}$ \\
\hline $\begin{array}{l}\text { User wants to refine } \\
\text { search (Exploration) }\end{array}$ & $\begin{array}{l}\text { Query on search } \\
\text { strategy }\end{array}$ & $\begin{array}{ll}- & \text { Expert response } \\
- & \text { Discussion of recent } \\
& \text { search history } \\
\end{array}$ & $\begin{array}{ll}- & \text { Email } \\
- & \text { Text/Audio/Video } \\
& \text { conference } \\
\end{array}$ \\
\hline $\begin{array}{l}\text { User doesn't get } \\
\text { expected response } \\
\text { from system }\end{array}$ & Query on why & $\begin{array}{ll}- & \text { FAQ } \\
\text { - } & \text { Expert response } \\
\text { - } & \text { Discussion of recent } \\
& \text { search history } \\
\end{array}$ & $\begin{array}{ll}\text { - } & \text { FAQ list } \\
\text { - } & \text { Email } \\
\text { - } & \text { Text/Audio/Video } \\
& \text { conference } \\
\end{array}$ \\
\hline
\end{tabular}

Table 1: Template for reference consultation genre systems.

At the present time, our observations are limited, and we do not know what specific genre systems will emerge as alternatives to the classic reference set. As experience is gained from running the pilot consultation service, we intend to elicit more information on how the expectations of a given genre are perceived by both librarians and users, how judgements are made by the former on issues such as the prioritisation of tasks, and how the latter perceive its effectiveness for solving their problems. At the beginning, when a common understanding of the relevance and consequences of genre characteristics is limited, patterns of use may be unpredictable, but we expect to see an 'ecology' of genre use evolving over time as both librarians and users learn to match consultation service genres against the particular constraints and requirements of recurrent situations. Yates, Orlikowski and Rennecke relate that in one of their studies, “... team members structured the genre systems themselves ..." instead of conforming with structures imposed by designers (Yates, Orlikowski and Rennecke, 1997: p. 57).

It is important for overall service quality and usability for both librarians and users to be able to determine the circumstances in which the use of specific genre would be most appropriate. We would also argue that it is important that librarians and users share a common understanding of how each employs genre, i.e., the assumptions and criteria upon which they base their selection of genre. As we have seen from the investigation, librarians respond to the cues available in the situation in which the query arises. The prototype system enhances these cues by enabling the sharing of search artefacts between librarian and user in any context. We have also seen how different genres are perceived by users to have distinctive characteristics. Refining 
the categories of the BIOSIS user survey, three genre characteristics would seem to be especially relevant to the users of network-based help services: effectiveness (capacity to deal with problems), availability (access opportunity), and responsiveness (speed of handling once initiated). We might tentatively match users' expectations of network reference consultation genres against these characteristics by drawing upon the res ults of the BIOSIS user questionnaires (see Table 2).

It is unclear what the implications are of ill-defined genre characteristics for users' genre selection. They may, for example, use worst-case assumptions (a possible set are shown in brackets in Table 2) as decision heuristics. It is easy to devise examples of where worst-case assumptions do not lead to optimum decisions, however. So, from the users' perspective, there is an argument for reducing uncertainty about genre characteristics. The problem is that unpredictability is situated, i.e., it arises in the specific context in which the query is received by the librarian. To reduce it, network reference consultation service users would either need more information about the librarian's circumstances at the time when the request is made, or service performance parameters must be clearly defined, i.e., "email queries will be answered with 24 hours". Either approach may pose problems for the librarians, however, if the result is that their capacity to prioritise their work is thereby reduced.

\begin{tabular}{|l|l|l|l|}
\hline Network Consultation & Effectiveness & Availability & Responsiveness \\
\hline $\begin{array}{l}\text { Synchronous: Text, } \\
\text { audio, video conference }\end{array}$ & High & $\begin{array}{l}\text { Unpredictable } \\
\text { (low) }\end{array}$ & High \\
\hline Asynchronous: email & Medium & High & $\begin{array}{l}\text { Unpredictable } \\
\text { (low) }\end{array}$ \\
\hline Asynchronous: FAQ list & $\begin{array}{l}\text { Unpredictable } \\
\text { (medium) }\end{array}$ & High & High \\
\hline
\end{tabular}

Table 2: Characteristics of genres for network reference consultations.

\section{Summary and Conclusions}

If users overall are making less use of expert's advice, but experts are still required to make them themselves available 'just-in-case', does this constitute a waste of highly salaried expert time? Some libraries appear to take this view and heavily restrict the hours when a professional reference service is available. Other libraries ret ain the presence of experts at a reference desk, but require them to catch up with cataloguing backlogs and other professional duties when not engaged in subject consultations. In cases where restricted professional service is unacceptable to users, a shared interinstitutional service with seamless remote consultation may be a solution, providing the infrastructure is in place. In cases where professional time is squandered sitting at the reference desk, remote intra-institutional consultation may also be acceptable. To promote understanding of how such remote reference services might work in the virtual library, we have offered an analysis of the activity which surrounds the reference consultation. 
Our preliminary investigation of collaboration between librarians and users of IR systems has presented a picture of a community that is in need of ways to reverse a growing fragmentation and the loss of those attributes that normally distinguish membership: relationships, mutual commitment, shared values and practices, shared artefacts and persistence (Erickson, 1997). A protoype network reference consultation system based upon the specific circumstances of this community is being implemented, and its use to support a pilot service will form the focus of future work.

Future research will combine analysis of genre systems with exploration of 'situated activity sequences', derived from the record of pilot service use. A growing archive of interactions (captured by online and offline logs) will allow us to establish what factors contribute to a positive outcome on a given occasion. From a design perspective, we suggest that this and the work of Erickson and others on genre analysis might contribute to the realisation of a usable and effective network reference consultation service.

\section{Acknowledgements}

The work reported here was funded by the Scottish Higher Education Funding Council, under their Regional Strategic Initiative programme. Ana Goldenberg is sponsored by CNPq (Brazilian National Research Council).

We would like to thank Margarete Tubby-Hille (Edinburgh University Data Library), Irene McGowan and David Carroll (Edinburgh University Library), Chris Atton (Napier University Library) and Catherine Fleming (Heriot-Watt University Library) for their participation, expertise and patience. We would also like to thank Sheila Cannell of Edinburgh University Library and Peter Burnhill, Director of EDINA (Edinburgh Data and INformation Access, Edinburgh University Library), whose support has been essential to the study.

Finally, we would like to thank the anonymous referees whose comments we found extremely helpful.

\section{References}

Ackerman, M. and McDonald, D. (1996) Answer Garden 2: Merging Organisational Memory with Collaborative Help. In Proceedings of CSCW'96. ACM Press, 97105.

Borgman, C. (1996) Why are online catalolgs still hard to use? Journal of the American Society for Information Science, 47(7), 493-503.

Brown, J.S. and Duguid, P. (1993) Stolen knowledge. Educational Technology, March, 10-15.

Brown, J.S. and Duguid, P. (1994) Borderline Issues: Social and Material Aspects of Design. Journal of Human-Computer Interaction, 9 (1), 3-36.

Crowston, K. and Williams, M. (1997) Reproduced and emergent genres of communication on the World-Wide Web. In Sprague, R.H. (Ed.), Proceedings of the thirtieth Hawaii International Conference on System Sciences, Volume VI. Washington: IEEE Computer Society Press, 30-39. 
East, H., Sheppard, E. and Yeal, Y. (1996) A huge leap forward -- a quantitative and qualitative examination of the development of access to database services by British universities 1988-1994. CCIS Policy Paper No 5. University of Westminster: CCIS, May.

Erickson, T. (1997) Social interaction on the Net: virtual community as participatory genre. In Sprague, R.H. (Ed.), Proceedings of the thirtieth Hawaii International Conference on System Sciences, Volume VI. Washington: IEEE Computer Society Press, 13-21.

Fleming, W.G. (1994) Methodography: the study of student learning as situated action. In Gibbs, G. (Ed.) Learning appproaches avaluation and strategy. Improivng Student learning through assessment and evaluation. Oxford: Oxford Brookes University, 525-544.

Gale, S. (1990) Human aspects of interactive multimedia communication. Interacting with Computers, 2, 175-189.

Hert, C. (1995) Searching as situated action. In ASIS 95: Proceedings of the 58th ASIS Annual meeting, Vol 32. Medford, NJ: Information Today Inc., 172-180.

Ingwersen, P. (1993) Information Retrieval Interaction. London: Taylor Graham.

Kuhlthau, C., Spink, A and Cool, C. (1992) Exploration into stages in the information search process in online information retrieval: communication between users and intermediaries. In Proceedings of the 55th Annual meeting of the American Society for Information Science. Medford, NJ: Learned Information, 67-71.

Kuhlthau, C. and Ledet, N. (1996) The Relation of Information and Uncertainty in Information Seeking. In Ingwersen, P. and Pors, N. (Eds) Proceedings of CoLIS 2: Second International Conference on Conceptions of Library and Information Science. Copenhagen: Royal School of Librarianship, 367-76.

Lave, J. and Wenger, E. (1991) Situated learning: legitimate peripheral participation. Cambridge: Cambridge University Press.

Marchionini, G. (1995) Information Seeking in Electronic Environments, Cambridge: Cambridge University Press.

McCarthy, J., Miles, V. and Monk, A. (1991) An Experimental Study of Common Ground in Text-Based Communication. In Proceedings of the Conference on Human Factors in Computing Systems, New Orleans, May. ACM Press, 209-215.

Olson, J., Olson, G. (1995) What Mix of Video and Audio is Useful for Small Groups Doing Remote Real-time Design Work. In Proceedings of the Conference on Human Factors in Computing Systems, Denver, May. ACM Press, 362-368.

Orlikowski, W. J. and Yates, J. (1994) Genre repertoire: norms and forms for work and interaction. MIT Sloan School Working Paper 3671-94; Centre for Coordination Science Technical Report 166, March. Cambridge MA: MIT.

Procter, R., Davenport, E., Burnhill, P. and Cannell, S. (1996) Providing Expert Assistance in the Virtual Library, Workshop on Information Retrieval and Human-Computer Interaction (GIST Technical Report G96-2). Glasgow, September.

Procter, R., Goldenberg, A., McKinlay, A. and Davenport, E. (1997) Enhancing Collaboration and Community in the Digital Library. In Proceedings of the $1^{\text {st }}$ European Conference on Digital Libraries, Pisa, September. Springer-Verlag.

Spink, A. and Saracevic, T. (1993) Dynamics of search term selection process in mediated online searching. In Proceedings of the 56th Annual Meeting of the American Society for Information Science. NJ: Learned Information, 63-72. 
Suchman, L. (1987) Plans and situated actions: the problem of human-machine communication. Cambridge: Cambridge University Press.

Taylor, R. S. (1968) Question-negotiation and information seeking in libraries. College and Research Libraries, 178-194.

Twidale, M. and Nichols, D. (1996) Collaborative browsing and visualisation of the search process. In Proceedings of ELVIRA-96, Milton Keynes, April.

Yates, J. and Orlikowski, W.J. (1994) Contextualizing Technology: From Ill-defined Borders to Socially Defined Genres. Journal of Human-Computer Interaction, 9 (1), 132-135.

Yates, J., Orlikowski, W.J. and Rennecker, J. (1997) Collaborative genres for collaboration: genre systems in digital media. In Sprague, R.H. (Ed.), Proceedings of the thirtieth Hawaii International Conference on System Sciences, Volume VI. Washington: IEEE Computer Society Press, 50-59.

Yates, J and Sumner, T. (1997) Digital Genres and the New Burden of Fixity. In Sprague, R.H. (Ed.), Proceedings of the thirtieth Hawaii International Conference on System Sciences, Volume VI. Washington: IEEE Computer Society Press, 312. 